\title{
Molecular Dynamics Simulation of Thermal Conductivity of Silicon Thin Film
}

\author{
Haitao Wang ${ }^{1}$, Yibin $\mathrm{Xu}^{1}$, Masato Shimono ${ }^{2}$, Yoshihisa Tanaka ${ }^{3}$ and Masayoshi Yamazaki ${ }^{1}$ \\ ${ }^{1}$ Materials Database Station, National Institute for Materials Science, Tokyo 153-0061, Japan \\ ${ }^{2}$ Computational Materials Science Center, National Institute for Materials Science, Tsukuba 305-0047, Japan \\ ${ }^{3}$ Composites and Coatings Center, National Institute for Materials Science, Tsukuba 305-0047, Japan
}

\begin{abstract}
We computed the thermal conductivity of silicon single crystal thin film with a thickness of $25 \mathrm{~nm}-134 \mathrm{~nm}$ at room temperature by nonequilibrium molecular dynamics simulation. The thermal conductivity was shown to depend on the thickness of the film, and is markedly lower than that in bulk silicon. The phonon classical thermal conductivity theory, incorporating the Boltzmann transport equation, was used to establish a phonon scattering model for size dependence. The results show that boundary scattering is very strong for phonon transport in silicon thin film. [doi:10.2320/matertrans.MAW200710]
\end{abstract}

(Received April 23, 2007; Accepted July 6, 2007; Published August 25, 2007)

Keywords: silicon thin film, thermal conductivity, molecular dynamics, Boltzmann transport equation, phonon boundary scattering

\section{Introduction}

Ultra-thin single crystal silicon films are becoming more common in microelectronics and optoelectronics systems. More needs to be known about the thermal behavior of ultrathin films, since significant heat flow can influence device performance and reliability. ${ }^{1,2)}$ Since semiconductor materials lack conducting electrons, their thermal conduction is dominated by phonons. Phonon behavior in thin films is different from that in bulk materials, and phonon scattering at the boundary will also affect thermal conduction, except for phonon-phonon scattering. Phonon boundary scattering is more important at low temperatures, where the phonon mean free path is longer. However, it may also be very significant at higher temperatures on a microscale comparable to the phonon mean free path. ${ }^{3)}$

Non-equilibrium molecular dynamics simulation (NEMD) is employed as a means for computing phonon thermal conductivity. ${ }^{4-8)}$ It introduces artificially hot and cold regions to induce heat flow. This heat flow creates a temperature gradient in the system that allows the thermal conductivity to be determined using Fourier's Law. The NEMD method is analogous to experimental conditions, and deals with the signal itself instead of its average fluctuations in an equilibrium state using the Green-Kubo method. As a result, it provides a more direct relation between lattice dynamics and thermodynamic properties. Schelling et al. ${ }^{9)}$ compared the non-equilibrium and equilibrium methods for monocrystalline silicon in detail, and obtained the thermal conductivity of bulk silicon by extrapolation to an infinite system size from a finite size, but the paper does not quantitatively demonstrate the mechanism by which phonon scattering is dependent on size.

In the present paper, a non-equilibrium molecular dynamics simulation was used to compute the thermal conductivity of single-crystal silicon thin film at room temperature. The Boltzmann transport equation was incorporated into the classical phonon thermal conductivity theory to quantify the size-dependent nature of phonon scattering.

\section{Simulation Methodology}

We used silicon single crystal with the long direction perpendicular to the (100) crystallographic plane as a model

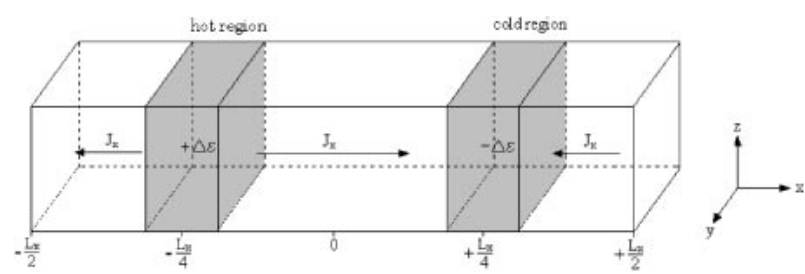

Fig. 1 Schematic diagram of three-dimensional simulation cell.

system. The equations of motion for all atoms in the system were integrated using a fifth-order Gear predictor-corrector algorithm with a time step of $0.5 \mathrm{fs}$. The Tersoff potential ${ }^{10)}$ was used to describe the silicon interatomic interaction in this simulation.

To compute the thermal conductivity of silicon single crystal, we constructed a three-dimensional simulation cell as shown in Fig. 1. The origin is located at the center of the cell. The hot and cold regions importing thermal disturbance are located at $x=-L x / 4$ and $x=+L x / 4$ so as to obtain a steady state heat flux and a temperature gradient. The hot and cold regions consisted of 10 atomic planes each, approximately $1.4 \mathrm{~nm}$ thick. The cross section of the simulation cell is equal to $5 \times 5$ silicon unit cells, approximately $7.4 \mathrm{~nm}^{2}$. The periodic boundary conditions are applied in all directions to form the silicon single crystal thin film. Heat $\Delta \varepsilon$ is added to the hot region, and the same amount of heat $\Delta \varepsilon$ is removed from the cold region at the same rates. We fixed $\Delta \varepsilon$ at approximately $5 \%$ of $k_{B} T$ at each time step, corresponding to $1.92 \times 10^{-22} \mathrm{~J}$ at room temperature. The energy modification is done by rescaling the atom velocities inside the hot and cold region,

$$
v_{\text {new }}=v_{\text {old }} \sqrt{1 \pm \frac{\Delta \varepsilon}{E}}
$$

where $v_{\text {old }}$ and $v_{\text {new }}$ are the atom velocities before and after rescaling, $\Delta \varepsilon$ is amount of energy added $(+)$ or removed $(-)$ during the time step, and $E$ is the total kinetic energy of the hot or cold region.

When the system achieves a steady state, the heat flux $J_{x}$ is given by

$$
J_{x}=\frac{\Delta \varepsilon}{2 A \Delta t}
$$




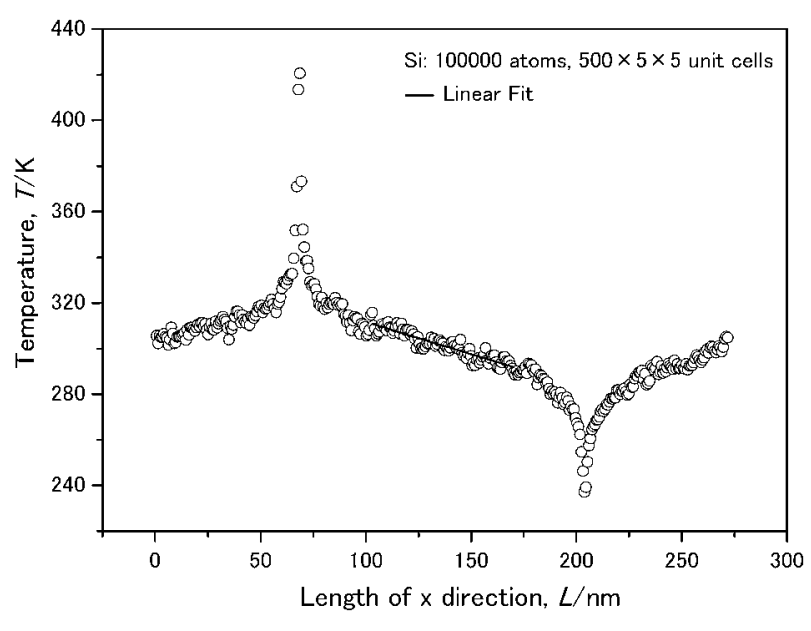

Fig. 2 Typical time-averaged temperature profile.

where $A$ is the cross sectional area perpendicular to the heat flux and $\Delta t$ is the time step. The factor of 2 arises from the periodic boundary conditions used here: energy flow from the hot region to the cold region in two directions.

We divided the simulation cell into a series of slices perpendicular to the $x$-axis to compute the temperature in each local area. Each slice includes approximately 250 atoms, which is sufficient for maintaining a local equilibrium. The local temperature is given by the Boltzmann statistic

$$
T_{j}=\frac{1}{3 k_{B} N_{j}}\left\langle\sum_{i=1}^{N_{j}} m_{i} v_{i}^{2}\right\rangle
$$

where $k_{B}$ is the Boltzmann constant, $N_{j}$ is the number of atoms in slice $j, m$ and $v$ are the mass and velocity of each atom, and \langle\rangle is the statistical average in the simulation time to avoid large temperature fluctuations. Since a heat flux is imposed and the temperature gradient is measured, the thermal conductivity can be given by Fourier's law. $\lambda=$ $J_{x} /(\partial T / \partial x)$.

\section{Results and Discussion}

Figure 2 shows the typical time-averaged temperature profile used to compute the thermal conductivity. This data was obtained using a $500 \times 5 \times 5$ silicon unit cell. To reach the steady state of heat flow, the system first runs 10,0000 time steps $(50 \mathrm{ps})$ to maintain equilibrium at room temperature in the NPT ensemble, then follows with long runs of 140,0000 time step (700 ps) with a heat flux of $2.61 \times$ $10^{10} \mathrm{~W} / \mathrm{m}^{2}$ are performed in the NVE ensemble. The nonlinear temperature profile is observed in slices near the hot and cold regions, which are attributed to the scattering caused by the hot and cold regions. In the intermediate region, the temperature profile is fitted with a linear relation. We therefore measured the temperature gradient about $34 \mathrm{~nm}$ away from the hot and cold regions.

Using the temperature distribution and heat flux, the thermal conductivity of silicon thin film of different thicknesses can be computed by Fourier's law, as shown in Fig. 3. The thickness of thin film ranges from $25 \mathrm{~nm}-134 \mathrm{~nm}$. The thermal conductivity was shown to depend on the thickness of the thin film, and is markedly lower than that in bulk

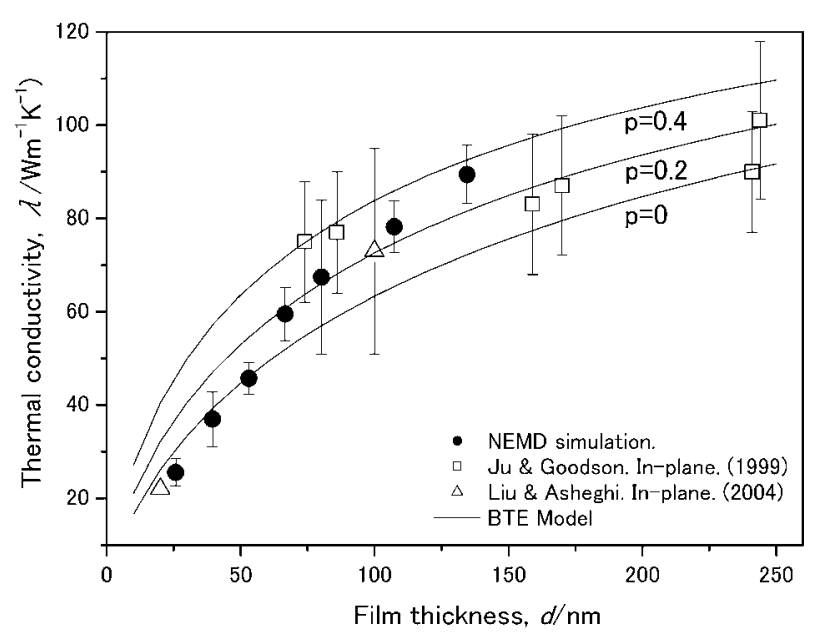

Fig. 3 Size dependence of thermal conductivity of silicon.

silicon $(148 \mathrm{~W} / \mathrm{mK})$.

To analyze this size dependence of silicon thin film, we considered the phonon classical thermal conductivity theory with the contribution of longitudinal and transverse acoustic phonon mode. The optical branch is sufficiently small to be neglected. ${ }^{3,11)}$

$$
\lambda=\frac{1}{3} \sum_{i=L, T 1, T 2} \int_{0}^{\frac{\Theta_{i}}{T}} C_{i}\left(x_{\omega}, T\right) \tau_{i}\left(x_{\omega}, T\right) v_{i}^{2} d x_{\omega}
$$

where the subscripts $i=L, T 1, T 2$ indicate the single longitudinal phonon mode and two transverse phonon modes; $x_{\omega}$ is the non-dimensional phonon frequency, $x_{\omega}=\hbar \omega /\left(k_{B} T\right)$, $\hbar$ and $k_{B}$ are Planck's constant divided by $2 \pi$ and the Boltzmann constant; $T$ is the system temperature; $\Theta_{i}$ is the Debye temperature; $C_{i}$ is the phonon specific heat per unit volume and unit non-dimensional frequency; $v_{i}$ is the phonon group velocity; and $\tau_{i}$ is the relaxation time per unit nondimensional frequency.

The phonon relaxation time of thin film $\tau_{i}$ includes phonon-phonon scattering and phonon boundary scattering, and can be given by

$$
\tau_{i}=\tau_{i, b u l k} F(\delta, p)
$$

where $\tau_{i, \text { bulk }}$ is the phonon relaxation time of the bulk material and $F(\delta, p)$ is the boundary scattering reduction factor.

The relaxation times of every phonon mode in bulk material are given as: ${ }^{11)}$

$$
\begin{aligned}
\tau_{L, \text { bulk }}{ }^{-1} & =B_{L} \omega T^{4} \\
\tau_{T, \text { bulk }}{ }^{-1} & =\tau_{T N, \text { bulk }}{ }^{-1}+\tau_{T U, b u l k}^{-1} \\
\tau_{T N, \text { bulk }}{ }^{-1} & =B_{T N} \omega T^{4} \\
\tau_{T U, \text { bulk }}{ }^{-1} & =0 \quad \omega<\omega_{1} \\
\tau_{T U, \text { bulk }}{ }^{-1} & =B_{T U} \omega T^{2} / \sinh x_{\omega} \quad \omega_{1}<\omega<\omega_{2}
\end{aligned}
$$

where $B_{L}, B_{T N}$ and $B_{T U}$ are constants.

The boundary scattering reduction factor $F$ can be obtained using the exact solution to the Boltzmann transport equation $^{12)}$

$$
F(\delta, p)=1-\frac{3(1-p)}{2 \delta} \int_{1}^{\infty}\left(\frac{1}{x^{3}}-\frac{1}{x^{5}}\right) \frac{1-e^{-\delta x}}{1-p e^{-\delta x}} d x
$$

where $\delta$ is the ratio of thin film thickness to phonon mean free 
Table 1 Parameters of silicon used in the calculation.

\begin{tabular}{llll}
\hline Parameters & Value & Parameters & Value \\
\hline$v_{L}$ & $8.48 \times 10^{3} \mathrm{~m} / \mathrm{s}^{11)}$ & $\Theta_{T U}$ & $210 \mathrm{~K}^{11)}$ \\
$v_{T}$ & $5.86 \times 10^{3} \mathrm{~m} / \mathrm{s}^{11)}$ & $\mathrm{B}_{L}$ & $2.0 \times 10^{-24} \mathrm{~s} / \mathrm{K}^{-311)}$ \\
$v_{T}\left(\omega>\omega_{1}\right)$ & $2.0 \times 10^{3} \mathrm{~m} / \mathrm{s}^{11)}$ & $\mathrm{B}_{T N}$ & $9.3 \times 10^{-13} \mathrm{~K}^{-311)}$ \\
$\Theta_{L}$ & $570 \mathrm{~K}^{11)}$ & $\mathrm{B}_{T U}$ & $5.5 \times 10^{-18} \mathrm{~s}^{11)}$ \\
$\Theta_{T N}$ & $180 \mathrm{~K}^{11)}$ & & \\
\hline
\end{tabular}

path of bulk material $\delta=d / l_{i, \text { bulk }} l_{i, b u l k}=\tau_{i, b u l k} v_{i} ; p$ is the specular reflection coefficient, which represents the probability of phonon specular reflection at the boundary.

The two transverse phonon modes are regarded as the same, and the three phonon mode contributions to the thermal conductivity of thin film are

$$
\begin{aligned}
\lambda & =\lambda_{L}+2\left(\lambda_{T N}+\lambda_{T U}\right) \\
\lambda_{L} & =\frac{1}{3} C_{L} T^{3} \int_{0}^{\frac{\Theta_{L}}{T}} \frac{x_{\omega}^{4} e^{x_{\omega}}}{\left(e^{x_{\omega}}-1\right)^{2}} \tau_{L, b u l k} F\left(\delta_{L}, p\right) d x_{\omega} \\
\lambda_{T N} & =\frac{1}{3} C_{T N} T^{3} \int_{0}^{\frac{\Theta_{T N}}{T}} \frac{x_{\omega}^{4} e^{x_{\omega}}}{\left(e^{x_{\omega}}-1\right)^{2}} \tau_{T N, \text { bulk }} F\left(\delta_{T N}, p\right) d x_{\omega} \\
\lambda_{T U} & =\frac{1}{3} C_{T U} T^{3} \int_{\frac{\Theta_{T N}}{T}}^{\frac{\Theta_{T U}}{T}} \frac{x_{\omega}^{4} e^{x_{\omega}}}{\left(e^{x_{\omega}}-1\right)^{2}} \tau_{T U, b u l k} F\left(\delta_{T U}, p\right) d x_{\omega}
\end{aligned}
$$

where $C_{i}=\left(k_{B} / 2 \pi^{2} v_{i}\right) /\left(k_{B} / \hbar\right)^{3}$.

The phonon classical thermal conductivity theory incorporating the Boltzmann transport equation makes the thermal conductivity of thin film a function of thickness and temperature. Phonon dispersion is critical in modeling the thermal conductivity of silicon near room temperature and above, where phonons with low group velocities become fully excited, ${ }^{13)}$ so the differential solution to the phonon frequency spectrum was used, and the boundary scattering reduction factor was independently computed for each frequency differential step. The parameters of silicon used in the model can be seen in Table 1 .

Figure 3 shows the result of the analytical model. $p$ is the probability of phonon specular reflection at the boundary, so $1-p$ means the degree of scattering at the boundary. When the thin film thickness is below about $60 \mathrm{~nm}$, there is $100 \%$ phonon scattering at the boundary. The degree of boundary scattering then falls with increasing thickness, but is still greater than $60 \%$ at about $130 \mathrm{~nm}$. It can be understood that the phonons have no chance to collide each other in less thickness, so all the phonons are scattered at the boundary. With increasing film thickness, the probability of phononphonon collision increases, and fewer phonons are scattered at the boundary. The experiment data in Fig. 3 come from the in-plane thermal conductivity of silicon monocrystalline thin films in Ref. 14) and 15). Although the experiment data is for in-plane thermal conductivity, it has close relevance to the NEMD simulation result for out-of-plane thermal conductivity in the present study.

\section{Conclusions}

A non-equilibrium molecular dynamics simulation was used to compute the out-of-plane thermal conductivity of monocrystalline silicon thin film with a thickness ranging from $25-134 \mathrm{~nm}$ at room temperature. The hot and cold regions of the simulation cell strongly scatter phonons, and a non-linear temperature profile was found near the hot and cold regions. However, the thermal conductivity can be obtained from the linear region based on Fourier's law. The size dependence of the thermal conductivity is very strong. Classical phonon thermal conductivity theory incorporating the Boltzmann transport equation can describe the relation between thermal conductivity, thickness of thin film, and temperature. The results show that boundary scattering is very strong in phonon transport within silicon thin film.

\section{Acknowledgements}

This study was financially supported by the Budget for Nuclear Research of the Ministry of Education, Culture, Sports, Science and Technology, based on the screening and counseling by the Atomic Energy Commission.

\section{REFERENCES}

1) D. G. Cahill, W. K. Ford, K. E. Goodson, G. D. Mahan, A. Majumdar, H. J. Maris, R. Merlin and S. R. Phillpot: J. Appl. Phys. 93 (2003) $793-$ 818.

2) A. D. McConnell and K. E. Goodson: Annual review of heat transfer. 14 (2005) 129-168.

3) M. Ashegi, Y. K. Leung, S. Wong and K. E. Goodson: Appl. Phys. Lett. 71 (1997) 1798-1800.

4) P. Jund and R. Jullien: Phys. Rev. B. 59 (1999) 13707-13711.

5) Y. G. Yoon, R. Car and D. J. Srolovitz: Phys. Rev. B. 70 (2004) 012302.

6) P. Heino: Phys. Rev. B. 71 (2005) 144302.

7) C. Oligschleger and J. C. Schon: Phys. Rev. B. 59 (1999) 4125-4133.

8) H. J. Castejon: J. Phys. Chem. B. 107 (2003) 826-828.

9) P. K. Schelling, S. R. Phillpot and P. Keblinski: Phys. Rev. B. 65 (2002) 144306.

10) J. Tersoff: Phys. Rev. Lett. 56 (1986) 632-635.

11) M. G. Holland: Phys. Rev. 132 (1963) 2461-2471.

12) E. H. Sondheimer: Adv. Phys. 1 (1952) 1-42.

13) G. Chen: Phys. Rev. B. 57 (1998) 14958-14973.

14) Y. S. Ju and K. E. Goodson: Appl. Phys. Lett. 74 (1999) 3005-3007.

15) W. Liu and M. Asheghi: Appl. Phys. Lett. 74 (2004) 3819-3821. 